\title{
Pedagogical Benefits from an Exercise in Reverse Engineering for an Aviation Software Systems
}

Emanuel S. Grant

University of North Dakota, emanuel.grant@UND.edu

Pann Ajjimaporn

How does access to this work benefit you? Let us know!

Follow this and additional works at: https://commons.und.edu/cs-fac

Part of the Computer Engineering Commons

\section{Recommended Citation}

Emanuel S. Grant and Pann Ajjimaporn. "Pedagogical Benefits from an Exercise in Reverse Engineering for an Aviation Software Systems" (2018). Computer Science Faculty Publications. 23.

https://commons.und.edu/cs-fac/23

This Conference Proceeding is brought to you for free and open access by the Department of Computer Science at UND Scholarly Commons. It has been accepted for inclusion in Computer Science Faculty Publications by an authorized administrator of UND Scholarly Commons. For more information, please contact und.commons@library.und.edu. 


\title{
Pedagogical Benefits from an Exercise in Reverse Engineering for an Aviation Software Systems
}

\author{
Emanuel S. Grant and Pann Ajjimaporn \\ Department of Computer Science, University of North Dakota, Grand Forks, U.S.A.
}

Keywords: $\quad$ Software Engineering, Reverse Engineering, Modelling Notation, UML, Activity Diagram, Safety-Critical Systems, Pedagogy, Curriculum.

Abstract: $\quad$ Since the Y2K crisis, reverse engineering has become a major area of work in industrial software application development, but lacks emphasis in US academia. This issue is exemplified by the high demand for software systems in new and expanding software application areas, which has resulted in systems being implemented before the requirements and design phases have been completed. Towards the maintenance of such systems, it is necessary to conducted reverse engineering for the derivation of software documentation for requirements and high-level and low-level design. When this scenario exists in the domain of safety-critical system, particularly in the aviation industry, reverse engineering takes on greater value because such software systems have to undergo development regulations and certification restrictions. This work reports on the pedagogical revelations gained from conducting reverse engineering on a software system that was developed and deployed for use in managing the assignment of commercial aircrafts to airport terminal gates. The software system incorporated genetic algorithms solutions and was implemented on a high-speed multi-processor system. The reverse engineering methodology applied was based on the RTCA DO-178C Software Considerations in Airborne Systems and Equipment Certification specification for onboard avionic software systems.

\section{INTRODUCTION}

In the last decades, there have been intense research activities in software development methodologies and modelling notations that have produced several notable ones, namely model-driven, componentbased, and agile methodologies, along with Coad/Yourdon (Coad, 1991), Shlaer/Mellor (Shlaer, 1988), and Unified Modelling Language (UML) (Glass, 1997) modelling notations. With each new methodology and modelling notation the goal has been an attempt to address the "software crisis" that was first identified in the late 1950s Booch, 1997). The software crisis is best defined as the inability of developers to deliver reliable software systems in a timely and cost-effective manner. This crisis is greater today than it has ever been, because of the increasing complexity and applications of software systems in many aspects of todays' business and personal endeavours.

The early proliferation of software development methodologies and notations did not resolve the situation, but exasperated it. Inter-project ventures were stymied by a project developers' unfamiliarity with the methodology and notation of another project. The problems arising from this over-growth of methodologies and notations were arrested with the merger of multiple modelling notations in a single representation the UML (Shlaer, 1988) and the methodologies coalescing around the Unified Process (Kruchten, 2003) methodology. The evolution and amalgamation of methodologies and notations, over the early 15 years are captured in Fig. 1, which was produced by Guido Zockoll, Axel Scheithauer \& Marcel Douwe Dekker. It should be noted that as of this date (first quarter 2018) the UML is at version 2.5 , sysML is at version $1.5, \mathrm{BPMN}$ is at version 2.0.2, and xUML is at version 1.1. These modelling notations have been developed by the Object Management Group (OMG) and the latest versions are not necessarily the ISO adapted version of the modelling notations.

Notwithstanding the availability of a de facto industry standard software modeling notation in the UML and accompanying methodologies such as the Rational Process, the software crisis is still an ev 


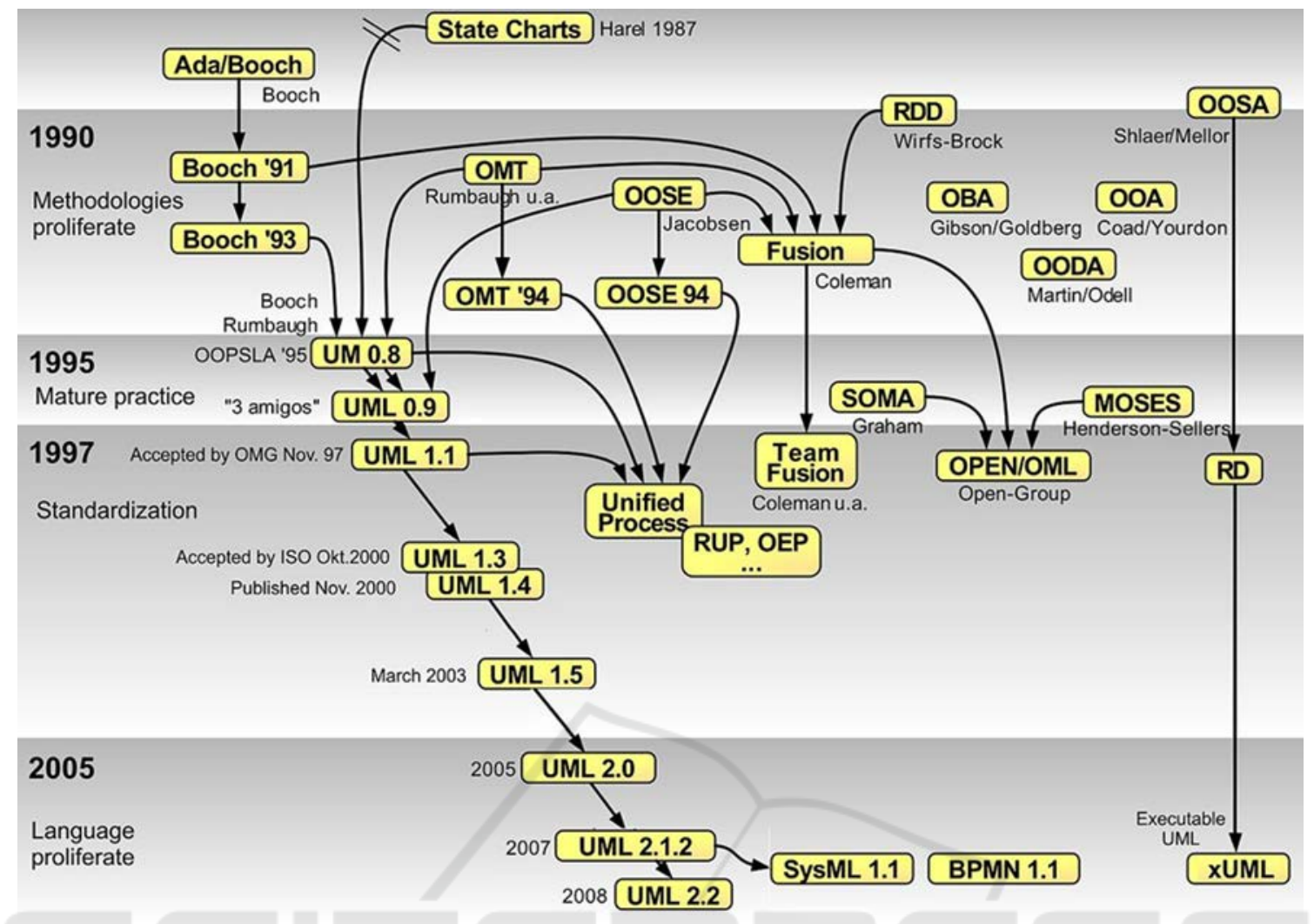

Figure 1: Evolution of object-oriented methods and notations 1980s - mid 2000s.

present phenomena of the software development industry. In the domain of safety-critical systems, the need to deliver correct and reliable software systems is at the highest priority. A daunting feature of safetycritical systems is the high degree of complexity in the design and implementation of such systems. Safety-critical software systems are characterized by the resulting loss or harm to life, if such systems fail during operation. Correspondingly, there is the associated domain of mission-critical software systems, wherein failure of those systems may result in significant damage to property and equipment. Examples of some of these safety-critical software systems' failure are the THERAC-25 (Leveson, 1993), the French Arian-5 rocket inaugural launch (Lions, 1996), and Air France flight 447 (AF447) of June 1, 2009 (Bureau, 2012). These failures overshadow the many successful applications of software systems in safety-critical environments, because of the high cost in property (Ariane-5 development cost US\$7 billion, payload US\$500 million), and lives (Air France 447, 216 passengers and 12 crewmembers).

Standards and methodologies play important roles in the development of safety-critical systems. Within the domain of avionic software systems, the RTCA organization has developed a standard, the DO-178C - Software Considerations in Airborne Systems and Equipment Certification (RTCA, 2011) for USA software development. A corresponding European EUROCAE ED-12C Software Considerations in Airborne Systems and Equipment Certification exist for avionic software development in the European territories. These documents set out a series of objectivities, activities, and data items that are required for the certification of on-board avionic systems. DO-178C is a revised standard of its previous version DO-178B, issued in late 2011 to incorporate new guidance regarding the use of objectoriented software development and the use of formal specification techniques in software development. The sole purpose of DO-178C is "for the production of software for airborne systems and equipment that performs its intended function with a level of confidence in safety that complies with airworthiness requirements” (RTCA, 2011). In order for a software system to be use onboard aircrafts in the USA, it has to be certified by the USA Federal Aviation Administration (FAA). The aviation software system must adhere to the DO-178C Specification and its supplemental specifications. 
In the USA, any of the software development methodologies, notations, and standards use in the industrial arena have come into use with relative independence of the curricula of tertiary software engineering programs. This situation results in a disconnect between the pedagogy of the classroom and the practices of the workplace. In the circumstance where there is collaboration on a software development project between academia and industrial partners there arise opportunities for academia to learn some of the concrete practices of the industry and then evolve the curricula to be more responsive to the desired skill-set of such program graduates. This report documents one such experience in the development of a software system to manage the assignment of commercial aircrafts to airport terminal gates with multiple and conflicting gate-assignment restrictions. The following sections of the manuscript documents the problem definition, in terms of the scope, deliverables, methodology, and pedagogy. The next section documents the experience of the project, followed by a discussion of the educational benefits derived from the project. The final section presents a summary in the form of a conclusion and a look at future work in this area.

\section{BACKGROUND}

\subsection{Problem Definition}

A commercial airline company, as a part of its operation review, identified a problem in its information system structure. The company encountered what was identified as a "single-point of failure" in the process for dynamic assignment of aircrafts to airport terminal gates. That single point of failure in the process was the reliance on a single specific operator to conduct dynamic assignment of aircrafts to terminal gates. The process involves the listing of all aircrafts for assignment and the available gates. Aircrafts are classified based on certain attributes, such as size, capacity, manufacturer, arrival time, departure time, etc. Gates are classified based on certain attributes, such as, location to runway, fuel-port, accessibility, availability time, etc. Other constraints pertain to global considerations, such as available runway, taxiway path to runway, established departure timeframes, etc.

The operator would compile the aircraft and gate lists and generate a standard assignment, based on the previous assignment cycle. The existing software system would then identify any assignment conflicts, which may arise from gate closures, incompatible aircraft-gate assignment, aircraft late or none arrival, etc. The operator would then attempt to resolve the assignment conflicts by reassigning aircrafts based on prior experience of executing this process. Whenever that operator is unavailable, the new operator would conduct the same operation, but the resolution would again be based on his experience.

The company recognized the failure that may arise if this system and process were not improved to be more efficient and effective. Consequently, a team of researchers from the University of North Dakota (UND) departments of Aviation and Computer Science were asked to look at the problem and develop a plan to mitigate the potentially problematic system and process. The UND team included researchers in genetic algorithm design and software engineering from the Department of Computer Science; it is the software engineering researchers' work, which is specifically documented in this report. Because of the nature of confidentiality and propriety information of the project, the airline will not be identified and information presented in the report has been sanitized.

\subsection{The Software Methodology}

The software development methodology applied on this project came out of the academic program taught in the university and research work on safety-critical system in general, and more specifically for avionic software systems. The genesis of the methodology was on an unmanned aerial system (UAS) for monitoring the flight operations of unmanned aerial vehicles (UAVs) in unrestricted airspace. In order to conduct software development in UAS domain the RTCA DO-178C specification was use as the definitive guideline. The work with DO-178C was two-fold: firstly, the document was transformed from its textual representation to a graphical representation, in the UML notation. Figures 2, and 3 illustrate two of the models developed to represent the DO-178C specification. Figure 2 represents the DO-178C specification, software development methodology components as an UML package-level model. Each package of Figure 2 is decomposed into a set of UML use case diagrams, class diagrams, and activity diagrams. Figure 3 represents the DO-178C Software Planning Process (Section 4 of the DO178C specification) as an UML Use Case Diagram, wherein the user is the project development team. Figure 3 is one of the models contained in the Software Planning Process 4.0 of the Figure 2 package-level model. 


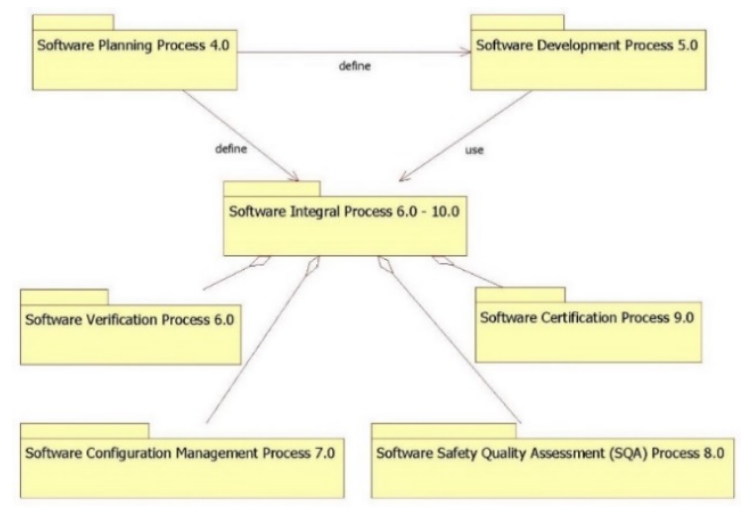

Figure 2: DO-168C UML Package-Level Representation.

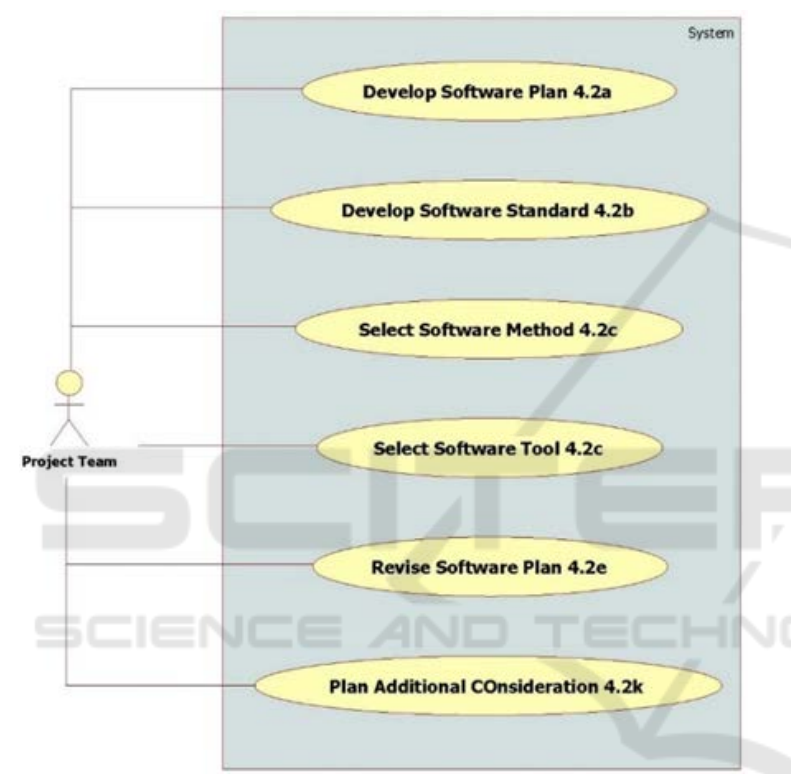

Figure 3: DO-178C Software Planning Process 4.0 UML Use Case Diagram Representation.

The second area of work with the DO-178C specification is the definition of a model-driven software development methodology that incorporates and is compliant with the DO-178C requirements. This methodology is illustrated in Figure. 4 in the form of an UML activity diagram. Figure 4 is an UML activity diagram representation of the requirement-level activities contained in the Software Development Process 5.0 package of Figure 2. The activities of Figure 4 are mapped to the respective sections of the DO-178C document, by way of the DO-178C section number being listed in the activities of the model. Figure 4 captures the activities as specified in the DO-178C for the software requirements analysis and design phases; the software implementation (coding), testing, and deployment phases are represented in separate UML activity diagrams. The work reported on in this manuscript is limited to the scope of Figure 4. The UML models specified in Figure. 4 are specific to this instantiation of the methodology; in other instantiations, other models may be used to satisfy the requirements of either the problem domain or the expertise of the development team.

A first task requirement of DO-178C Software Planning Process (4.0) is the determination of the software level of development. DO-178C specifies five (5) levels of criticality, designated Level-A through Level-E, with Level-A being the highest and Level E the lowest. Once the software level has been determined then DO-178C Software Development Process (5.0) and Software Integral process (6.0 10.0) specify the required set of activities and data element necessary for certification of the system that is to be developed. The outputs of these activities are the Software Plan (4.2a), Software Standard (4.2b), Software Method (4.2c), and Software Tool (4.2c), as listed in Figure 3. There may be Additional Considerations, for the particular application domain.

\subsection{The Pedagogy}

A version of the Figure 4 methodology is included in the curriculum of the software engineering undergraduate course and a graduate-level formal specification course in the computer science course at UND. In the undergraduate-level course, students are taught a number of software development methodologies, and are required to develop a small software system by using a minimal version of the methodology of Figure 4. In a similar manner at the graduate-level, the students are required to develop a more complex system than that of the undergraduatelevel. An additional requirement, at the graduatelevel, is that the system is assumed to be at the DO178C criticality Level-A, thus necessitating the use of rigorous system validation and verification $(\mathrm{V} \& \mathrm{~V})$ techniques as an activity of the used development methodology. These V\&V activities are executed at the Verify Low Level Design 6.3 and Verify Low Level Design 6.3, of Figure 4. This formal specification technique involves the derivation of $\mathrm{Z}$ notation (Potter, 1996) representation of the UML models that were developed of the system at the activities of Conduct High Level Design 5.2.2 and Conduct Low Level Design 5.2.2 of Fig. 4. 


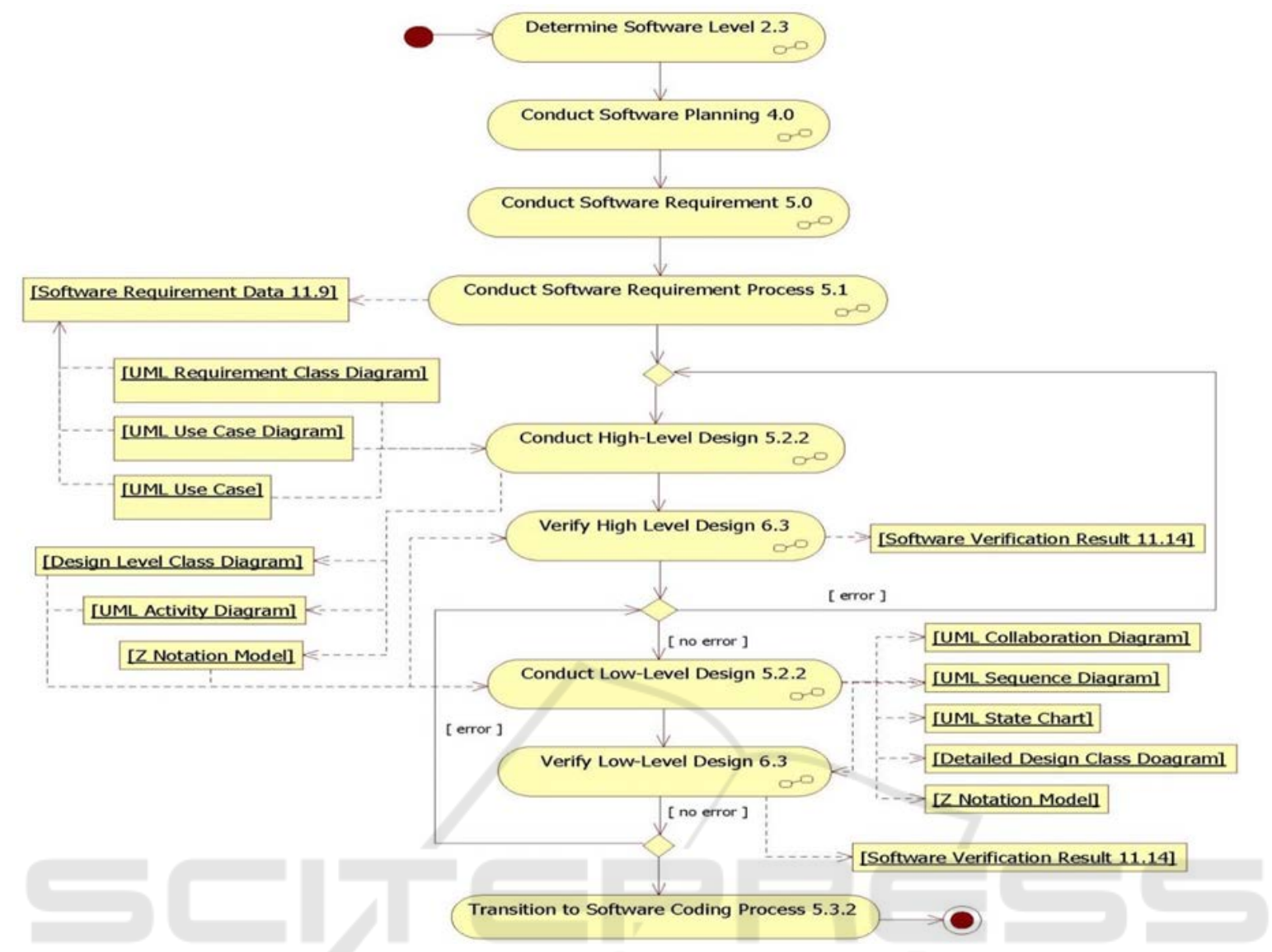

Figure 4: UML Activity Diagram Representation of DO-178C Compliant Model-Driven Methodology.

The software engineering courses are taught once per year and follow a strict forward engineering methodology approach. The topic of reverse engineering is covered toward the end of the teaching cycle, subject to the availability of lecture time; teaching time on higher priority topics may be extended thus reducing time for the lower priority topics.

A review of the software engineering programs at ten major universities in the USA, did not encounter the term "reverse engineering" as ether a topic or course in any of the listings. Search of the catalogue of a major USA academic publishing firm for textbooks on reverse engineering produced a hit list of four items; similar searches for the terms "requirement engineering”, "software design" and "code generation" produces kit lists close to or exceeding 100 items. These observations imply that the topic of reverse engineering is not as widely taught and written about in academia as are the other phases of the software development life cycle.

\section{PROJECT DESCRIPTION}

At the start of the project, the Department of Computer Science researchers formed three teams. One team focused on developing the genetic algorithms to implement the aircraft-to-gate assignment solution. The second team focused on the design and implementation of the user interface of the system. The third team focused on the documentation of the system, by way of modelling and verification/validation exercises. The teams are hereinafter referred to as Team I, Team II, and Team III respectively. The content of this report is a documentation of the efforts of Team III on project. Notwithstanding the fact that the teams worked independent of each other, to a great degree, there was a high level of integration between the teams, as Team II worked on the interface to the genetic algorithms and Team III developed models of both systems for verification and validation, and system documentation. A secondary goal of Team III was the identification and capture of any pedagogical 
principles for incorporation into the curricula of software engineering courses, taught by the Department of Computer Science.

The teams held joint and separate interviews with the airline's stakeholders; namely managers, system administrators, and operators over the life of the project, and typically had greater number of meetings at the start and end of the project. Meetings at the start of the project were geared towards capturing the full requirements of the system, while meetings towards the end of the project were targeted at system verification and acceptance. At the initial phase of the project, the teams sought to establish a common set of system requirements, coming out of their respective independent and joint meetings with the stakeholders. Once these requirements were finalized, the teams progressed at different rates of work during the early stages of the project. In joint meetings between the teams, Team III determined that their initial models of the system were not synchronized with the work products of the other two teams, as there were supplemental meetings with some of the stakeholders and some requirements had been modified, eliminated, or new ones introduced. This realization led to Team III reorganizing their standard approach to the model development activities.

\subsection{Modified Methodology}

Research showed that many software development projects fail because of the inability to deliver the product in a timely and cost effective manner, i.e. the software crisis. Paul Dorsey list ten reasons why systems projects fail (Dorsey, 1998). Among the Dorsey's list is the lack of use of an appropriate software development methodology and focusing the development efforts on coding. Teams I and II had initiated what may be best described as an Agile approach to developing the system's user interface and generic algorithm solutions, as they rapidly produce coded components of the system. The teams refined the code, after consultation with the stakeholders, towards having a working system at the earliest.

Team III ascertained that the initial strategy for modelling the system would not be successful; hence, the team modified the development methodology in use to accommodate the work of the other two teams. This modification was an iterative reverse engineering process that is illustrated in Figure 5

The process model of Figure 5 was developed to incorporate a reverse-engineering strategy to complete the forward-engineering activities. This process model also illustrates the use of formal specification techniques for validating the reverse and forward engineering activities. The "Design UML Models" activity of Figure 5 is reflective of the Conduct High Level Design 5.2.2" and "Conduct Low-Level Design 5.2.2" of Figure. 4, and the "Formal Models" activity of Figure 5 is synonymous to the "Verify High Level Design 6.3" and "Verify Low-Level Design 6.3" activities of Figure 4. The green (solid) arrowed lines represent the forward engineering path through the process model, while the red (broken) arrowed lines represent the reverse engineering path through the model. The forward engineering process commenced with the "Design UML Models" activities, while the reverse engineering process commenced at the "Program Code" activity.

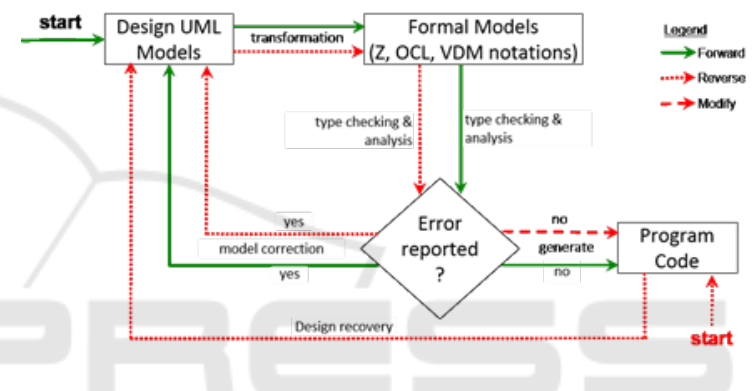

Figure 5: Reverse-Engineering Modified Model-Driven Methodology.

This modification to the development methodology then transitioned along the reverse engineering line "Design recovery" line, from the "Program Code" to representative "Design (high and low) UML Models”. The UML models were then transformed to a formal representation in the $\mathrm{Z}$ notation for analysis during the verification phases of the Figure 4 methodology. If the models pass the verification, then work transition along the "generate" arrowed lines to the production of "Program Code". Otherwise, work transition along the "model correction" arrowed line to the UML models and the next iteration of the process commence with the identified errors being corrected in the models.

\subsection{Project Implementation}

Team I developed an acceptable generic algorithm solution for the airline-gate assignment problem by pursuing an Agile based methodology. The team held monthly meetings with the airline's stakeholder managers and operators to present the accomplished goals and establish a new set of goals for the next 
scheduled meeting. Visits were alternated between the airline's operation facility and UND research labs. Each iteration resulted in the refinement of achieved goals, accomplishment of established goals, or the definition of new goals. While these sprints were unusually long for a Scrum framework, they proved adequate for this particular domain, because of the complexity of the requirements and the development strategy. Shorter sprints would have produced incomplete goals at the level of granularity that would be understandable to the stakeholders. Team I was successful in completing a genetic algorithm application system that was acceptable to the stakeholders. The software system emulated the actions of resolving aircraft-gate assignments in an optimal manner that was equal to or better than that which the experience operator could devise. This ensured that even in the absence of an operator the aircraft-gate assignment conflict resolution would be completed in a timely manner for the airlines operations.

Team II's effort to develop a user interface for the gate-assignment conflict resolution system, was simplified after it was determined that the existing user interface had to undergo minor modifications to accommodate the new system. The modifications involved adding a menu item for executing the aircraft-gate assignment conflict-resolution system. Consequently, the modelling of the user interface system was not conducted by Team III, as the existing documentation for the user interface was assessed to be sufficient for the airlines system administrators.

\subsection{Team Iii Efforts}

Team III effort was centred on that of reverse engineering a set of UML models of the genetic algorithm system for the purpose of verification, validation, and system documentation. Team III opted to identify this system as a Level-A DO-178C system, in order to exercise as many of the modeldriven methodology's activities, as represented in Figure 4. The intent was to garner as much pedagogical benefits as possible for incorporation into the software engineering curricula of the department and provide comprehensive system documentation artefact to the stakeholders.

The main UML model developed by Team III was a set of activity diagrams that was implemented at the detailed-level of system modelling. The limitation to producing just one type of UML model was borne out of the airline system administrators' preference for just the necessary models to facilitate any immediate small-scale bug fixes, versus models to be used for system evolution. The nature of the contract between UND and the airline called for the software system's on-going maintenance (evolution) to be further contracted out to a third party.

A sanitized example of a segment of one of the UML activity diagrams that was developed is presented in Figure. 6. Figure 6 does not illustrate any significantly unusual activity diagram modelling technique, but with the exception of the listing of some activities with generic titles, such as "Activity 1", “Activity 2", etc.

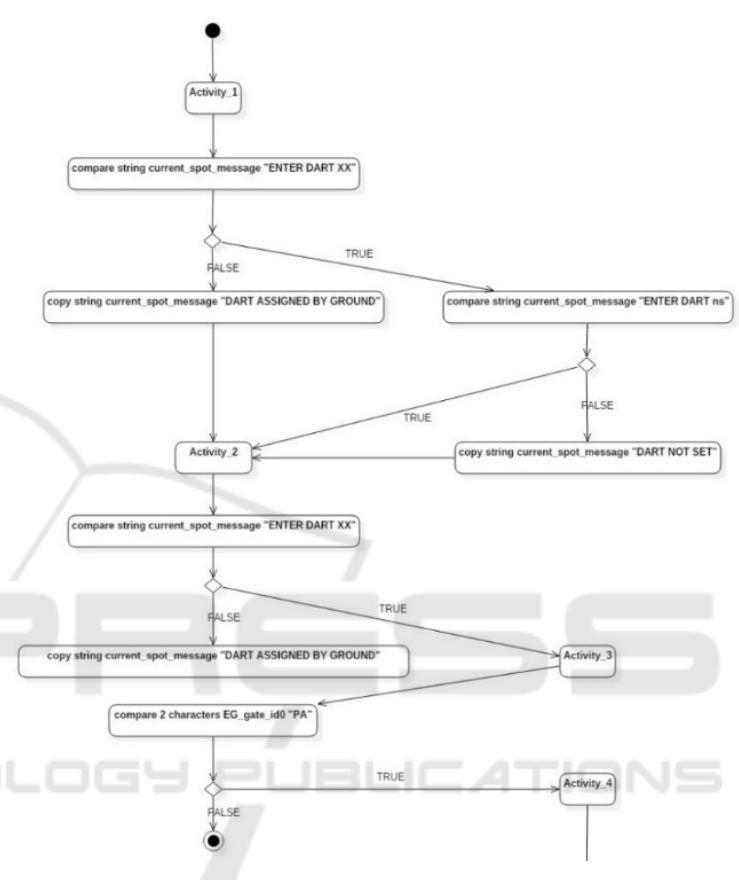

Figure 6: UML Activity Diagram of Aircraft-Gate Assignment System.

This was done in order to capture very low-level details of the program code, from which the model was reversed engineered.

The models were developed in the open-source tool StarUML and the contents of "Activity-Xs" were stored in the documentation fields of the models. As implemented in this activity diagram, "Activity 2 " is the snippet of code presented in Figure 7

Team I did not implement a fully object-oriented programming paradigm, but partitioned the codeproduction exercise into modules based on four phases of the operation; (1) list aircrafts and terminal gates, (2) assign aircrafts to gates, (3) generate conflicts, and (4) resolve conflicts. Team III completed the reverse engineering of the models with the assistance of graduate students in the department, under the supervision of faculty researchers. The work was completed over one and one half years. 
Team III's work was partitioned into two phases; the first phase covers the reverse engineering of the UML activity diagram models, and the second, future, phase will involve the formal verification of the models.

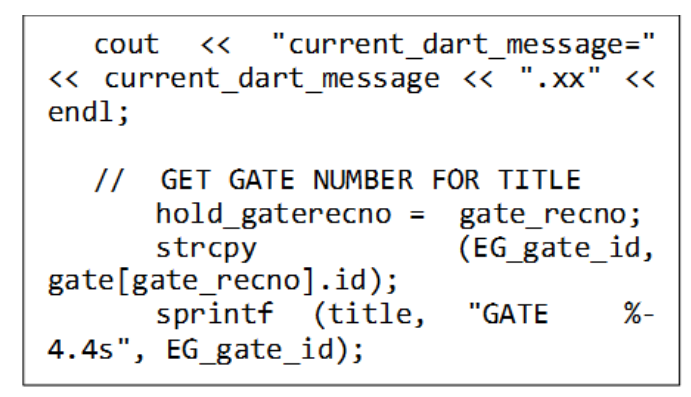

Figure 7: Code Snippet from UML Activity Diagram.

\section{RESULT}

Software system performance must always be deterministic in the domain of safety-critical systems. These software systems encompass numerous highly complex processing components and have high demands for reliability and accuracy, in order to safeguard against failure.

Because of the extensive use of UML in software development, there is a need to restate the informal semantics of the models produces. Transforming UML models into $Z$ equivalent schemas provides formal analysis to accomplish verification and validation of software systems Clachar, 2010). With the ever-growing demand for software systems in existing, new, and emerging application areas, strict requirements and design phase activities of software development methodologies are sometimes not enforced. Consequently, industrial practices incorporate reverse engineering as a necessary phase of software system development. This is done in order to capture the necessary software system modelling artefacts for system documentation and maintenance.

In many USA universities, reverse engineering is normally taught as an "add-on" to software development methodologies. The resulting situation is that graduates leave these software engineering programs with minimal knowledge about reverse engineering then find themselves in a work environment where reverse engineering is of paramount importance. The experience of the faculty researchers on the project documented in this report, and from a prior project on the development of an UAS airworthiness system for monitoring UAVs operation in a restricted airspace, is that there needs to be a change in the pedagogical approach to teaching reverse engineering.

The student researchers on this project and a prior project, in which reverse engineering was also applied, expressed specific and strong opinions on the need to be taught formal approaches to reverse engineering. Some of these student researchers had participated in internship programs at a variety of industrial organizations and had been exposed to reverse engineering tasks. There was a unanimous conclusion that reverse engineering is important to the software development activities in real-world project and consequently, they think the process should be offered in courses on the same level as forward engineering topics. There was also a consensus among the student researchers that working with code at the start of the project was challenging and this challenge may be alleviated if they had grounding in techniques to re-construct the code.

The faculty researchers are now revising the curricula of two undergraduate-levels and one graduate-level courses in software engineering, at the UND Department of Computer Science, to address this identified disconnect between industrial practice and the pedagogy. The curricula revision is multifaceted, with changes in lecture content, assignments, and project requirements at both the undergraduate and graduate levels of teaching. Selection of chapters and articles from textbooks and journals on reverse engineering will be listed for reference reading to both groups of students, with some being selected as required reading for each of the two groups. It should be noted that while there is an abundance of textbooks on forward engineering (requirements engineering, software design, and software implementation) there are less known and available textbooks on reverse engineering, which are suitable for academia. Assignments and projects will now include specific work on reverse engineering in a form that is based on the experience from the aforementioned two projects. Critical to the new reverse engineering pedagogy will be an emphasis on Agile software development methodologies as a class of methodologies that fosters the incorporation of reverse engineering techniques.

\subsection{The Revised Curriculum}

The revised software engineering curriculum will continue to be project-based, but will now include activities in reverse engineering. The software engineering methodology of instruction for the course 
will be based on the work defined in figures 4 and 5 of this report and the specific pedagogical topics covered are influenced from the experience on the aforementioned projects. Specifically, students will be introduced to the relationship between forward and reverse engineering, as illustrated in Figure 5. Reverse engineering topics to be covered in the revised curriculum include, but are not limited to the following:

- Use of CASE tools in reverse engineering. Specifically, open source tools will be used, example StarUML, so that students can work on their one computers.

- Reverse engineering techniques for objectoriented programming versus procedural programming.

- Techniques to manually reverse engineer program codes that include: identifying methods' names, methods' inputs and outputs, and call sequences between methods.

- Techniques to identify programming constructs, such as: assignments, iterations, decisions, selections, etc.

- Techniques for transforming programming code into pseudo-code.

- Techniques for transforming pseudo-code into graphical models, namely UML models.

The teaching strategy applied in the revised curriculum will have the students working in teams to develop a moderately complex system as a forward engineering exercise. Concurrently, the teams will work on reverse engineering the code of a wellknown simple textbook system, such as the library management system (Singh 2010). Both project will be preceded by lectures on the fundamental principles of software engineering, and concurrent lectures on detailed and supplemental software engineering topics. Pre- and post-surveys to determine the students' comprehension of the relationship between academia and professional software engineering learning and practices will be conducted. The data from these surveys will aid in improving the curriculum.

\section{CONCLUSIONS}

This report documents the experience gained from a collaborative project between academia and industry for developing a mission-critical software system, albeit, the system was assessed as a safety-critical application for educational purposes. The project was conducted by teams of USA academic faculty and student researchers, in difference spheres of focus. One team conducted a reverse engineering exercise in order to develop a set of graphical UML models, from the program code of the system. These models formed the main artefacts of documentation and verification of the software system. This team had an adjacent project goal of identifying aspects of the project that would be incorporated in the curricula of software engineering courses.

The project successfully achieved the established goal by providing a software system to the stakeholders that was introduced into production within the specified timeframe. The adjacent project goals of identifying pedagogical benefits from the project were realized, as the hypothesis of a knowledge gap existence between the curricula of some USA undergraduate and graduate tertiary software engineering education and industrial practices was exemplified and data collected to address this issue. The outcome is that the curricula of these aforementioned courses have been revised to include the teaching of reverse engineering as a firstclass topic of the courses under review. Future work will seek to evaluate the benefits of this revised pedagogy to the productivity of the graduates from the courses. Future alumni surveys will include specific questions to assess these benefits.

\section{REFERENCES}

Coad, P., Yourdon, E., 1991. Object-Oriented Design, Prentice Hall, Inc. New Jersey, USA.

Shlaer, S., Mellor, S. J., 1988. Object Oriented Systems Analysis: Modeling the World in Data, $1^{\text {st }}$ ed., Prentice Hall, New Jersey, USA.

Glass, R. L., 1997. The Software-Research Crisis, IEEE Software, IEEE Computer Society Press, California, USA, vol. 11. No. 6, pp, 42-47.

Booch, G., Rumbaugh, J., Jacobson, I., 1997. The Unified Modeling Language, Rational Software Corporation, Addison-Wesley, Indiana, USA.

Kruchten, P. 2003 The Rational Unified Process: An Introduction, $3^{\text {rd }}$ ed., Addison-Wesley Object Technologies Series, Indiana, USA.

Leveson, N. G., Turner, C. S., 1993. An Investigation of the Therac-25 Accidents, IEEE Computer, IEEE Computer Society, vol. 26, No. 7, pp 18-41.

Lions, J., 1996 ARIANE 5, Flight 501 Failure, Report by the Inquiry Board, European Space Agency, Paris, France.

Bureau d'Enquêtes et d'Analyses, 2012, Final Report on the Accident on 1st June 2009 to the Airbus A330-203 Registered F-GZCP operated by Air France flight AF 447 Rio de Janeiro - Paris, Bureau d'Enquetes et d'Analyses France (BEA), Paris, France. 
RTCA, 2011. Software Considerations in Airborne Systems and Equipment Certification. DO-178C, Radio Technical Commission for Aeronautics (RTCA), Washington DC, USA.

Potter, B., Sinclair, J., Till, D., 1996. An Introduction to Formal Specification and Z, $2^{\text {nd }}$ ed., Prentice Hall Europe, Hertfordshire, UK.

Clachar, S., Grant, E. S., 2010. A Case Study in Formalizing UML Software Models of Safety Critical Systems, The Annual International Conference on Software Engineering. Global Science and Technology Forum (GSTF), Phuket, Thailand.

Dorsey, P., 1998. 10 Reasons Why Systems Projects Fail, Technical Report, Dulcian, Inc.

Singh, D., 2010, C++ Library Management System Project - Source Code of Program, CPPforschool C++ Tutorial for School Students, Ghaziabad, India. 